\title{
Multidrug-resistant tuberculosis around the world: what progress has been made?
}

Dennis Falzon, Fuad Mirzayev, Fraser Wares, Inés Garcia Baena, Matteo Zignol, Nguyen Linh, Karin Weyer, Ernesto Jaramillo, Katherine Floyd and Mario Raviglione

Affiliation: Global TB Programme, World Health Organization, Geneva, Switzerland.

Correspondence: Dennis Falzon, Global TB Programme, World Health Organization, 20 avenue Appia, CH1211, Geneva 27, Switzerland. E-mail: falzondawho.int

ABSTRACT Multidrug-resistant tuberculosis (MDR-TB) (resistance to at least isoniazid and rifampicin) will influence the future of global TB control. $88 \%$ of estimated MDR-TB cases occur in middle- or highincome countries, and 60\% occur in Brazil, China, India, the Russian Federation and South Africa.

The World Health Organization collects country data annually to monitor the response to MDR-TB. Notification, treatment enrolment and outcome data were summarised for 30 countries, accounting for $>90 \%$ of the estimated MDR-TB cases among notified TB cases worldwide.

In 2012, a median of $14 \%$ (interquartile range 6-50\%) of estimated MDR-TB cases were notified in the 30 countries studied. In 15 of the 30 countries, the number of patients treated for MDR-TB in 2012 (71 681) was $>50 \%$ higher than in 2011. Median treatment success was 53\% (interquartile range $40-70 \%$ ) in the 25 countries reporting data for 30021 MDR-TB cases who started treatment in 2010.

Although progress has been noted in the expansion of MDR-TB care, urgent efforts are required in order to provide wider access to diagnosis and treatment in most countries with the highest burden of MDR-TB.

@ERSpublications

A wider adoption of effective diagnostics, treatment and preventive measures is needed to control MDR-TB globally http://ow.ly/zwZWr

This article has supplementary material available from erj.ersjournals.com

Received: June 042014 | Accepted after revision: June 302014 | First published online: Sept 262014

The authors alone are responsible for the views expressed in this publication and they do not necessarily represent the decisions or policies of the World Health Organization (WHO). The designations used and the presentation of the material in this publication do not imply the expression of any opinion whatsoever on the part of WHO concerning the legal status of any country, territory, city or area, or of its authorities, nor concerning the delimitation of its frontiers or boundaries.

Support statement: All authors are staff members of the World Health Organization (WHO). The United States Agency for International Development (USAID) was a principal salary supporter of the WHO staff involved in this article.

Conflict of interest: None declared.

The content of this work is @World Health Organization. Design and branding are @ERS 2015. ERJ Open articles are open access and distributed under the terms of the Creative Commons Attribution Non-Commercial Licence 4.0. 


\section{Introduction}

In 2012, the World Health Organization (WHO) estimated that $~ 5.7 \%$ of incident tuberculosis (TB) cases worldwide had multidrug-resistant TB (MDR-TB) (resistance to at least isoniazid and rifampicin) [1]. Although this appears to be relatively low, MDR-TB cases pose a disproportionate threat to the global prospects of $\mathrm{TB}$ control and are expensive to diagnose and treat [2,3]. In several eastern European countries, at least one-third of TB cases presenting for treatment have MDR-TB $[4,5]$. In a number of countries across the world, population rates of MDR-TB cases have increased over time [6, 7]. Of the 1.3 million TB deaths in 2012, 170 000 (13\%) were estimated to be among people with MDR-TB. MDR-TB treatment remains arduous and unsatisfactory: whereas $87 \%$ of all newly notified TB patients reported to WHO complete their medication successfully, only about one half of MDR-TB cases do so and the likelihood of success diminishes with progressive resistance beyond MDR-TB [8-10]. To date, 100 countries worldwide have reported at least one case of extensively drug-resistant TB (XDR-TB) (MDR-TB plus resistance to any fluoroquinolone and any second-line injectable drug) [11].

In line with a 2009 World Health Assembly (WHA) Resolution [12], the Global TB Programme of WHO monitors the efforts made by countries to expand universal access to MDR-TB care and prevention. In this study, we use the latest data reported to WHO by early 2014 to summarise recent efforts being made to detect and cure MDR-TB patients in countries with the highest caseload of drug-resistant TB. We suggest interventions that could accelerate the progress being made.

\section{Materials and methods}

Each year, countries report aggregated national data on standard TB indicators to WHO via a web-based system. These reports are used by WHO to monitor TB worldwide, and for MDR-TB specifically, to monitor progress with respect to the targets established in "The Global Plan to Stop TB 2011-2015", which were themselves aligned to the 2009 WHA resolution (table 1) [13]. The surveillance definitions and parameters have been standardised for several years and the calculation of these indicators has been described elsewhere [14-16].

Data were analysed for the 30 countries with $>1000$ estimated MDR-TB cases among notified pulmonary TB patients in 2012 (fig. S1 of the online supplementary material). The MDR-TB cases in these 30 countries together account for $>90 \%$ of the estimated global MDR-TB caseload. Data reported by these countries were used to describe: 1) the coverage of drug-susceptibility testing (DST) for isoniazid and rifampicin (conventional or molecular) among TB cases, and for fluoroquinolones and second-line injectable drugs among MDR-TB cases; 2) MDR-TB and XDR-TB case notifications; 3) new enrolment on MDR-TB treatment; and 4) MDR-TB treatment success and coverage of treatment-outcome reports. Time trends for notification and enrolment indicators (2009-2012) and treatment outcome cohorts (2007-2010) were also analysed. Expenditure data for second-line drugs, as reported to WHO, were used to describe the relative contributions of domestic (government and loan) and external (Global Fund and other donors) support for MDR-TB diagnosis and treatment by country. The country income groups used are those defined by the World Bank in 2013 [17].

The estimates of MDR-TB cases for each country were derived by multiplying the latest available value for the proportion of TB cases with MDR-TB by the total number of pulmonary TB cases notified in 2012 (the methods and individual country estimates are provided in further detail elsewhere [1]. The number of MDR-TB cases actually notified was compared with the estimated number of MDR-TB cases to assess case detection. Additional cases with rifampicin-resistant strains detected using Xpert MTB/RIF (Cepheid, Sunnyvale, CA, USA) who would also be eligible for MDR-TB treatment were also reported by some countries and these were included alongside the MDR-TB cases in our analyses. The ratio of cases enrolled on MDR-TB treatment to the number of MDR-TB cases detected was used to express the degree of

TABLE 1 Key multidrug-resistant tuberculosis (MDR-TB) indicators and targets for 2015

New bacteriologically positive TB patients tested for resistance to first-line drugs $\quad 20 \%$

Previously treated TB patients tested for resistance to first-line drugs

Countries reporting $>50 \%$ of the MDR-TB cases that are expected to exist among notified TB cases $100 \%$

Confirmed MDR-TB cases commencing treatment in programmes that follow international $\quad 100 \%$ guidelines

Treatment success among patients with confirmed MDR-TB

Countries reporting treatment outcomes for all confirmed MDR-TB cases

The indicators and targets listed are from "The Global Plan to Stop TB 2011-2015" [13]. 
alignment between the diagnostic and curative capacities of national health systems. Treatment outcomes were reported 24 months after the end of the year of observation [15]; we therefore present results for patients who commenced treatment in 2010. The number of cases in which outcome was monitored (excluding the cases that were not evaluated) was compared with the number of MDR-TB cases originally notified in 2010 in order to assess the coverage of treatment outcome reporting. The de-notification of cases that were later found not to have MDR-TB may account for the differences between the number of patients originally notified and those reported with an outcome. As the reasons for these discrepancies are not always reported to $\mathrm{WHO}$, the percentage outcomes were computed using the number of cases who are reported with an outcome as part of an annual cohort.

When summarising values across the different countries, the central tendency and spread of the data are primarily expressed as medians and interquartile ranges (IQR), respectively. The mean annual percentage change was used to express the overall change in detection and enrolment between 2010 and 2012; this statistic was not calculated when data were missing for any of the 3 years considered. All statistics were generated using $\mathrm{R}$ and all figures were created using the ggplot2 (www.ggplot2.org) package working in the same environment ( $\mathrm{R}$ version 3.0.1) $[18,19]$.

\section{Results}

Global distribution of the MDR-TB burden

The 30 countries that were included accounted for an estimated total of 280000 MDR-TB cases (95\% uncertainty range $190000-330000)$ of the global total of 300000 MDR-TB cases that are estimated to exist among notified pulmonary TB cases (table 2). India, China, the Russian Federation, the Philippines, Pakistan and South Africa had, in that order, the highest number of MDR-TB patients, and they accounted for more than two-thirds of the cases from the 30 countries.

\section{Coverage of DST}

19 countries provided data for 2012 on the coverage of DST for isoniazid and rifampicin among new patients (median 1.6\% (IQR 0.1-78.1\%)) (fig. 1a). In previously treated TB patients, DST coverage also ranged widely among the 22 countries providing data $(10.6 \%(2.1-46.3 \%))$. Seven eastern European countries and Peru reported DST results for $>20 \%$ of new patients, and Belarus and Kazakhstan reported $>80 \%$ of previously treated cases with DST results. The targets of the "The Global Plan to Stop TB 20112015 " are $20 \%$ in new and $100 \%$ in previously treated TB cases (table 1 [13]. In the 15 countries with data for 2012, the coverage of DST for both fluoroquinolones and second-line injectable drugs among MDR-TB patients was 43.0 (15.5-69.5) (data by country not shown).

\section{MDR-TB and XDR-TB notifications}

In 2012, the case detection of MDR-TB varied between countries from $<1 \%$ and $>100 \%$ of the estimated caseload (median 14.4\% (IQR 5.8-50.2\%)) (fig. S2 of the online supplementary material). Eight countries (Belarus, Kazakhstan, Kyrgyzstan, Peru, the Republic of Korea, the Republic of Moldova, South Africa and the Ukraine) detected $>50 \%$ of their estimated cases, which is the target threshold for all countries by 2015. In 2012, a total of 77956 MDR-TB cases was detected plus an additional 8986 rifampicin-resistant TB cases detected using Xpert MTB/RIF alone, a doubling in reported cases in the 30 countries studied compared with 2009 (table 2 and fig. 2). Overall, the mean annual percentage increase was 27\% during 2010-2012, but exceeded 100\% in Angola, India, Myanmar, Nigeria, Pakistan and Viet Nam. However, MDR-TB case detection in 2012 was $<10 \%$ of the estimated number in Angola, Nigeria and Viet Nam. 15 of the 30 countries reported at least one XDR-TB case in 2012; out of the total 2077 XDR-TB cases reported, 1596 (77\%) were reported by South Africa alone (data by country not shown).

\section{Enrolment on MDR-TB treatment}

The number of patients commencing MDR-TB treatment increased overall between 2009 and 2012. There were successive annual increments in new enrolments between 2010 and 2012 in at least 18 countries, and an increase of $>50 \%$ in 2012 was seen in 15 countries, in comparison with 2011 (table 2 and fig. 2). Overall, the mean annual percentage increase in enrolments was 34\% between 2010 and 2012; this was $>100 \%$ in Angola, Belarus, India, Nigeria, the Philippines and Viet Nam. In 2012, the ratio of patients enrolled on treatment to the number of MDR-TB cases detected had a median value of 77.9\% (59.5$101.3 \%$ ); the target for 2015 is $100 \%$ in all countries. The ratio at times exceeded $100 \%$, usually as a result of the enrolment of cases notified in a previous year or without laboratory confirmation (considered further in the Discussion). 
TABLE 2 Multidrug-resistant tuberculosis (MDR-TB) cases estimated, detected and commencing treatment: the top 30 countries

\begin{tabular}{|c|c|c|c|c|c|c|c|c|c|}
\hline \multirow[t]{2}{*}{ Country } & \multirow[t]{2}{*}{ Best estimate 2012 n (95\% CI) } & \multicolumn{4}{|c|}{ Cases detected and reported } & \multicolumn{4}{|c|}{ Cases commencing treatment } \\
\hline & & 2009 & 2010 & 2011 & $2012 \mathrm{n}$ (\% of estimated) & 2009 & 2010 & 2011 & 2012 \\
\hline Afghanistan & $1100(0-2900)^{\#}$ & 0 & 19 & 19 & $31(3)$ & 0 & 0 & 21 & 38 \\
\hline Angola & $1700(780-2500)^{\#}$ & 0 & 3 & 40 & $45(3)$ & 0 & 3 & 5 & 45 \\
\hline Azerbaijan & $2800(2600-3000)$ & 0 & 552 & 811 & $596(21)$ & 0 & 286 & 592 & 406 \\
\hline Bangladesh & 4200 (3100-5200) & 0 & 339 & 509 & $513(12)$ & 352 & 339 & 390 & 513 \\
\hline Belarus & 2200 (2100-2200) & 1342 & 1576 & 1594 & 1604 (73) & 0 & 200 & 1446 & 2478 \\
\hline Brazil & 1700 (1400-2000) & 449 & 573 & 566 & $684(40)$ & 398 & 573 & 630 & 713 \\
\hline China & 59000 (52 000-66 000) & 474 & 2792 & 1601 & 3007 (5) & 458 & 1222 & 1155 & 1906 \\
\hline DPR Korea & $3800(3000-4600)^{\#}$ & 0 & 0 & 37 & $25(1)$ & 0 & 0 & 25 & 50 \\
\hline DR Congo & $2900(670-5100)^{\#}$ & 91 & 87 & 121 & 81 (3) & 176 & 191 & 128 & 262 \\
\hline Ethiopia & 2000 (1200-2900) & 233 & 140 & 212 & $284(14)$ & 88 & 120 & 199 & 289 \\
\hline India & 64000 (49 000-79 000) & 1660 & 2967 & 4237 & $16588(26)$ & 1136 & 2967 & 3384 & 14143 \\
\hline Indonesia & 6900 (5200-8500) & 0 & 182 & 383 & $428(6)$ & 20 & 142 & 260 & 426 \\
\hline Kazakhstan & 7000 (6900-7200) & 3644 & 7387 & 7408 & 7608 (>100) & 3209 & 5705 & 5261 & 7213 \\
\hline Kenya & $2800(840-4800)^{\#}$ & 150 & 112 & 166 & $225(8)$ & 140 & 118 & 156 & 202 \\
\hline Kyrgyzstan & 1800 (1600-2000) & 785 & 566 & 806 & 958 (53) & 545 & 566 & 492 & 775 \\
\hline Rep. Moldova & 1700 (1600-1800) & 1069 & 1082 & 1001 & 894 (53) & 334 & 791 & 765 & 853 \\
\hline Mozambique & 2000 (1300-2700) & 140 & 165 & 283 & $266(13)$ & 103 & 87 & 146 & 213 \\
\hline Myanmar & $6000(4600-7500)$ & 815 & 192 & 690 & $778(13)$ & 64 & 192 & 163 & 442 \\
\hline Nigeria & $3600(2700-4500)$ & 28 & 21 & 95 & $107(3)$ & 0 & 23 & 38 & 125 \\
\hline Pakistan & $11000(0-29000)^{\#}$ & 49 & 444 & 344 & $1602(15)$ & 368 & 424 & 344 & 1045 \\
\hline Peru & $2200(2100-2300)$ & 1578 & 1048 & 1663 & $1225(56)$ & 1856 & 1702 & 1374 & 1510 \\
\hline Philippines & 12000 (9300-15 000) & 1073 & 522 & 1148 & $679(6)$ & 501 & 548 & 2397 & 1918 \\
\hline Papua New Guinea & $1100(930-1300)^{\#}$ & 0 & 0 & 15 & $58(5)$ & 0 & 0 & 60 & 82 \\
\hline Republic of Korea & 2200 (1800-2700) & 0 & 450 & 516 & 1212 (55) & 0 & 0 & 307 & 1212 \\
\hline Russian Federation & 46000 (43000-49000) & 14686 & 13692 & 13785 & $13612(30)$ & 8143 & 13692 & 18902 & 18452 \\
\hline South Africa & 8100 (6900-9400) & 9070 & 7386 & 10085 & 15419 (>100) & 4143 & 5402 & 5643 & 6494 \\
\hline Thailand & 1800 (1400-2200) & 0 & 0 & 510 & $492(27)$ & 296 & 9 & 123 & 0 \\
\hline Ukraine & $6800(6500-7000)$ & 3482 & 5336 & 4305 & $6934(>100)$ & 3186 & 3870 & 4957 & 7672 \\
\hline Uzbekistan & 4000 (3700-4300) & 654 & 1023 & 1385 & $1728(43)$ & 464 & 628 & 855 & 1491 \\
\hline Viet Nam & $3800(3000-4600)$ & 217 & 101 & 601 & 273 (7) & 307 & 101 & 578 & 713 \\
\hline Total & 280000 (190 000-330 000) & 41689 & 48757 & 54936 & 77956 (28) & 26287 & 39901 & 50796 & 71681 \\
\hline
\end{tabular}

Data are presented as $n$, unless otherwise stated. The \% detected may exceed $100 \%$ if estimates were too conservative, and if linkage between clinical and laboratory registers was inadequate. Further information can be found in the Materials and methods section. ${ }^{\#}$ : based on modelled values from countries in the same region. 


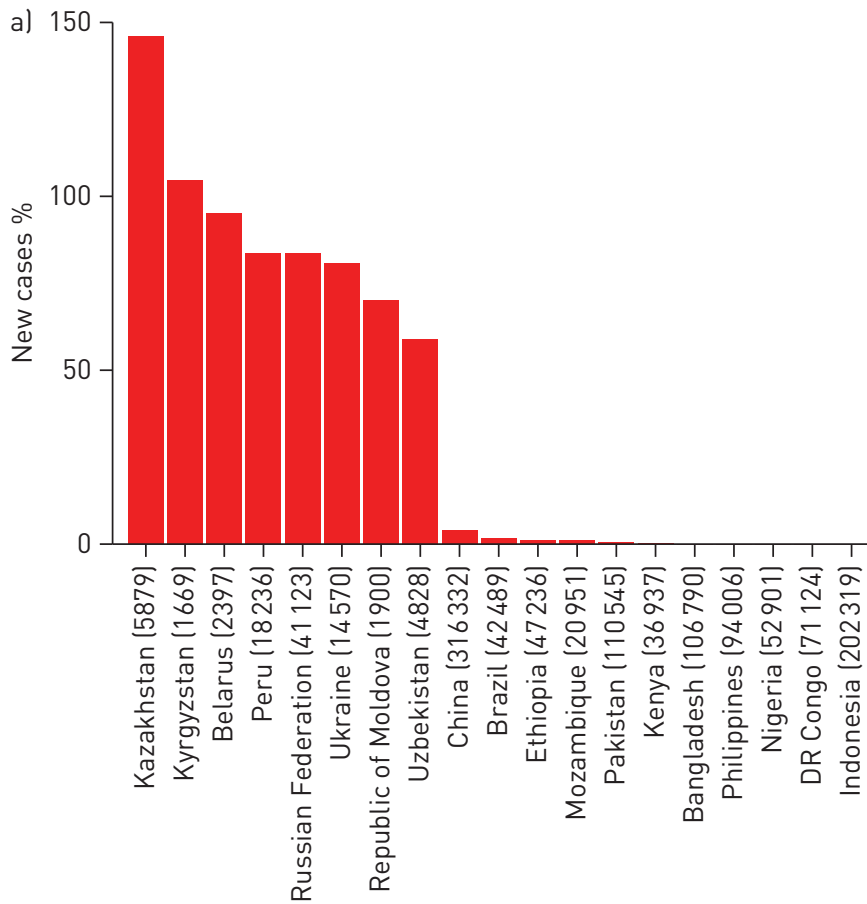

Country (cases notified $n$ )

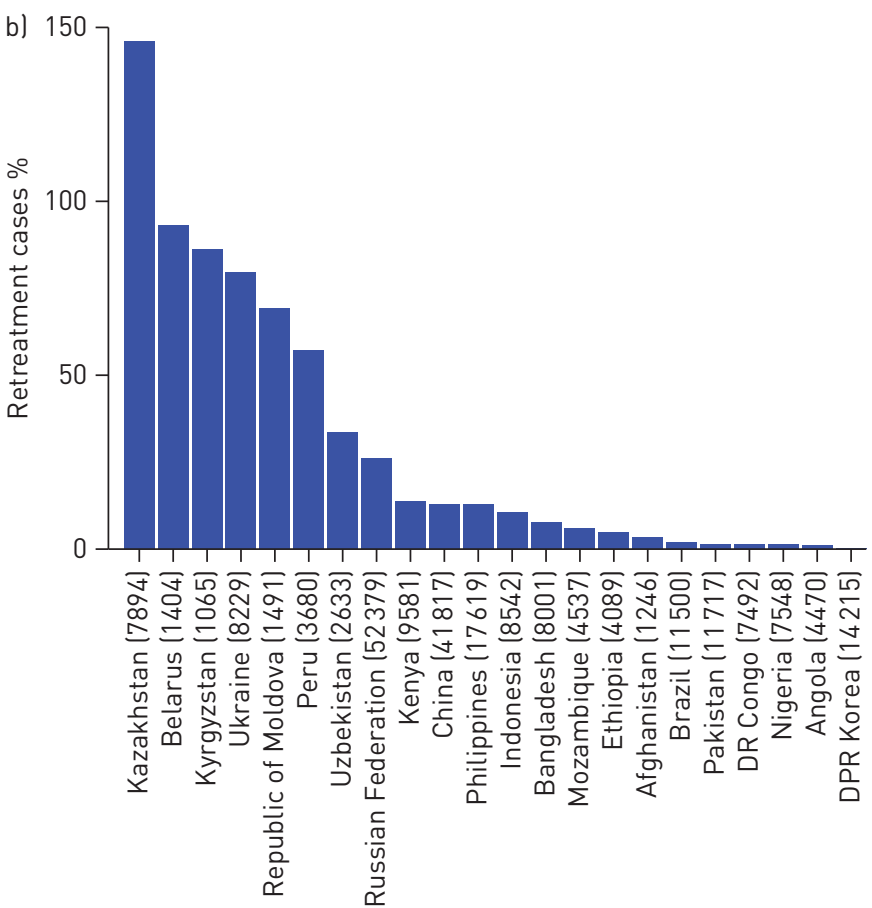

Country (cases notified $\mathrm{n}$ )

FIGURE 1 a) New and b) retreatment tuberculosis cases with drug-susceptibility test results for isoniazid and rifampicin, 2012, by country. Inadequate linkage between clinical and laboratory registers may result in an overestimation of drug-susceptibility testing coverage (e.g. $>100 \%$ in Kazakhstan).

\section{Treatment success and coverage of treatment outcome reporting}

25 of the 30 countries reported treatment outcomes for MDR-TB patients who began treatment in 2010. The median treatment success was 53\% (IQR 40-70\%), and in four countries (Bangladesh, Ethiopia, Kenya and Viet Nam) it ranged from $75 \%$ to $84 \%$, thus achieving the target of $\geq 75 \%$ in "The Global Plan to Stop TB 2011-2015" [13]. The success ratio has been largely stable in most countries but did increase in Brazil and Peru from 2007 to 2010, alongside improvements in the completeness of data (fig. 3). Deaths represented $>25 \%$ of the 2010 cohort in Nigeria, Papua New Guinea and the Ukraine, while the sum of cases lost to follow-up or not evaluated exceeded $40 \%$ in Belarus, the Democratic Republic of Congo, India, Mozambique and the Philippines.

The number of MDR-TB cases monitored for outcome increased over time in more than half of the countries. The median proportion of MDR-TB cases notified in 2010 for whom outcome data were reported was $74.4 \%$ (IQR $52.7-87.0 \%$ ), with nine countries reaching $\geq 80 \%$ of the MDR-TB patients beginning treatment, including the four reaching the treatment success target in 2010 (fig. S3 of online supplementary material). Countries are expected to achieve 100\% outcome reporting coverage by 2015.

\section{Country income groups and expenditure on second-line drugs}

Only nine countries (accounting together for $\sim 10 \%$ of the total estimated cases among the 30 countries) were in the low-income group (table 3); almost 75\% of the estimated caseload was from the 19 middle-income countries and $17 \%$ was from the two high-income countries (Republic of Korea and the Russian Federation). The distribution of notified MDR-TB cases mirrored that of estimated cases, with $4 \%$ in low-income, $77 \%$ in middle-income and $19 \%$ in high-income countries.

Expenditure data for second-line drugs were provided by 12 countries, accounting for $58 \%$ of the estimated MDR-TB cases in the 30 countries; in addition, Thailand reported data for the Bangkok metropolitan administration alone (fig. 4). The total funding received by the programmes and spent on these drugs amounted to US $\$ 192$ million in $2012 ; 27 \%$ of this funding was from external sources. The proportion of external funding had no clear association with the number of MDR-TB cases notified by these countries. The share of external funding was inversely associated with gross national income per capita, adjusted for purchasing power parity until $\sim$ US $\$ 5000$ per capita (fig. S4 of the online supplementary material). In the Russian Federation (the only high-income country in the group that reported expenditure data), spending on second-line drugs originated wholly from domestic sources. In 

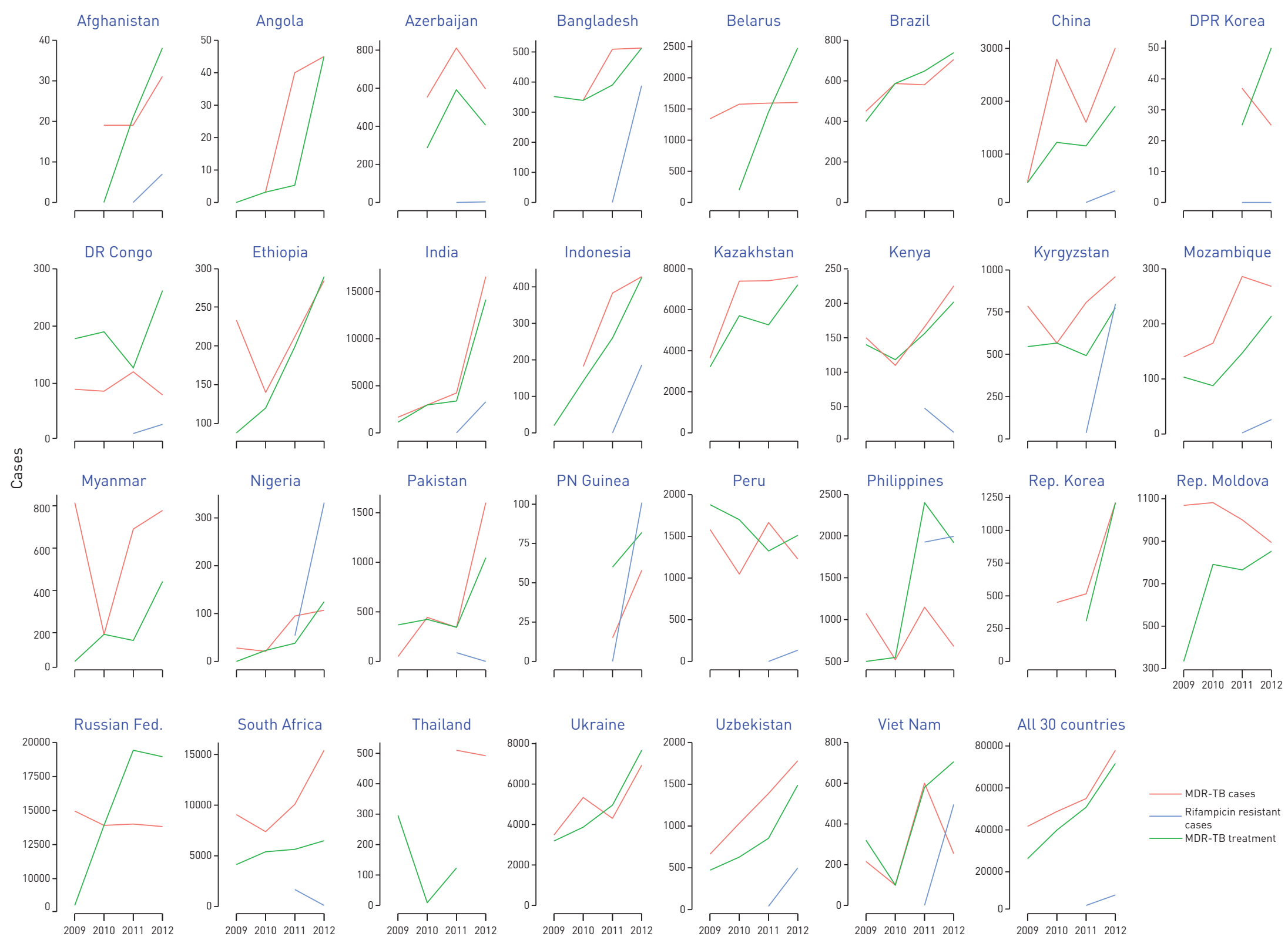

FIGURE 2 Multidrug-resistant tuberculosis (MDR-TB) cases and additional rifampicin-resistant TB cases detected, compared with TB cases enrolled on MDR-TB treatment, trend in target countries, 


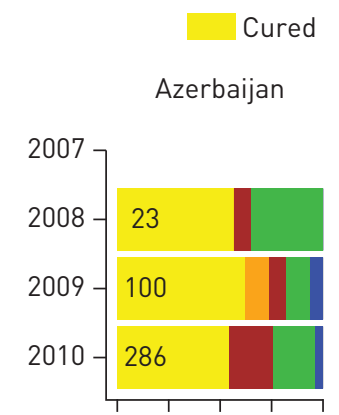

Ethiopia
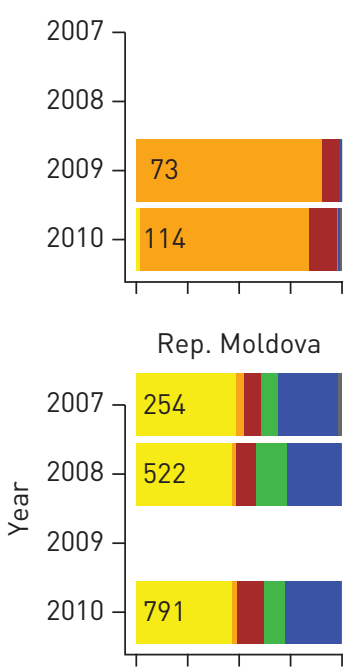

Philippines

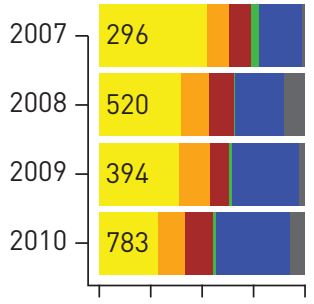

South Africa

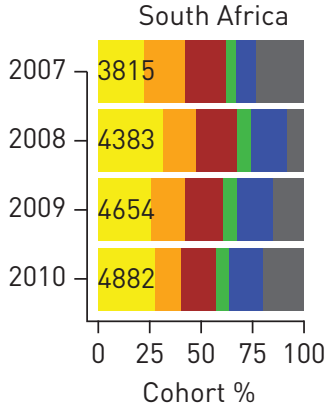

Completed

Bangladesh

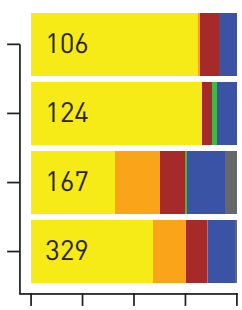

Indonesia

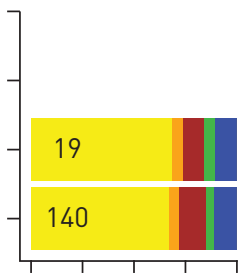

Myanmar

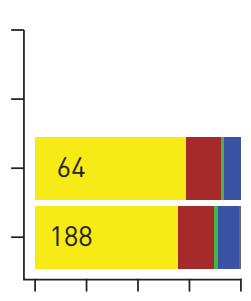

PN Guinea

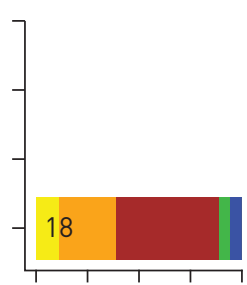

All 30 countries

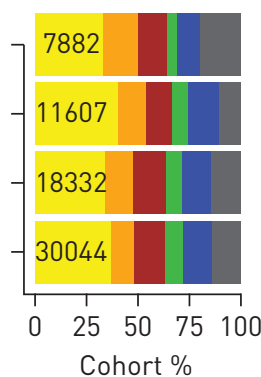

Died

Belarus

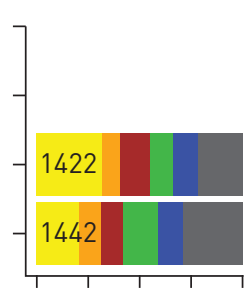

India

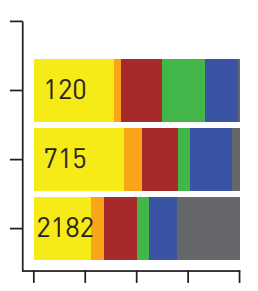

Mozambique

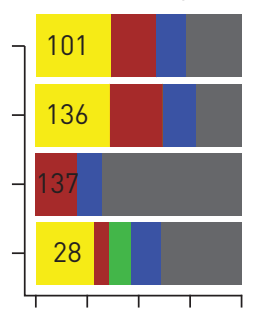

Russian Fed.

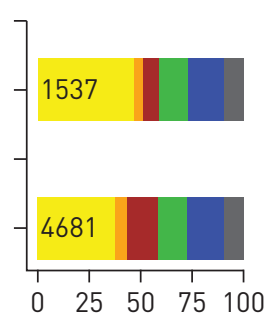

Cohort \%
Brazil

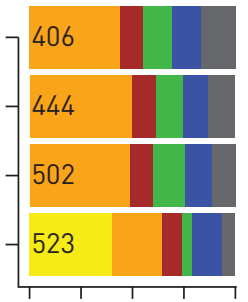

Kazakhstan

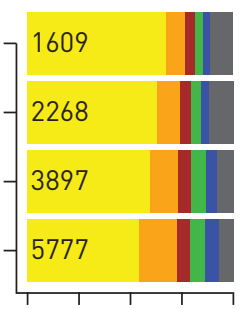

Nigeria

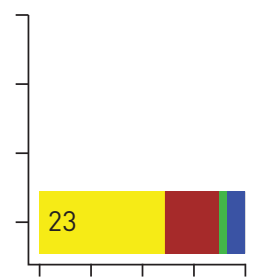

Ukraine

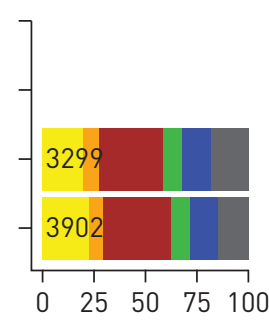

Cohort \%
China

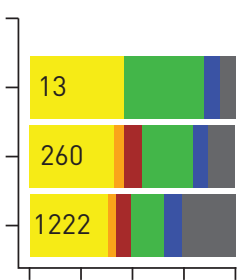

Kenya

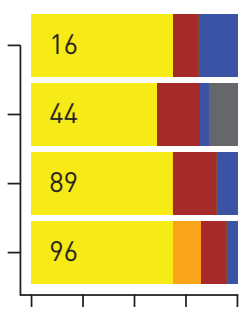

Pakistan

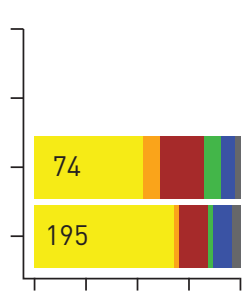

Uzbekistan

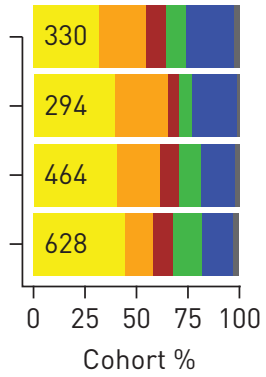

DR Congo

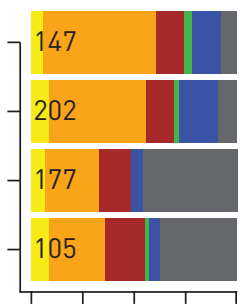

Kyrgyzstan

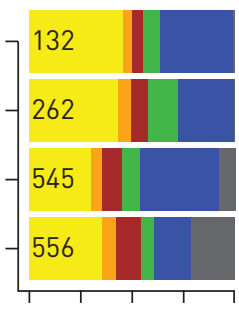

Peru

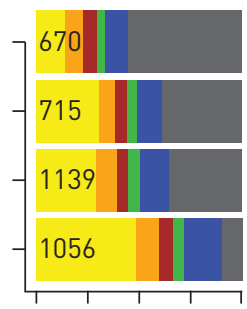

Viet Nam

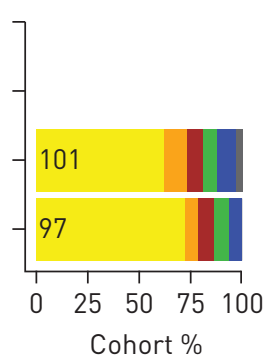

FIGURE 3 Treatment outcomes for patients diagnosed with multidrug-resistant tuberculosis by target country, 2007-2010 cohorts. The total number of cases in each annual cohort is shown on the bars.

contrast, in the two low and five lower middle-income countries, second-line drugs were entirely supported by external grants. In upper middle-income countries, the proportion of funding received for second-line drugs from external sources varied, and was $>50 \%$ in Belarus and China. 
TABLE 3 Number of estimated and notified multidrug-resistant tuberculosis (MDR-TB) cases by country income group, 2012

Income group

Top 30 countries

\begin{tabular}{cc}
\hline Countries $n$ & $\begin{array}{c}\% \text { of countries by } \\
\text { income group }\end{array}$
\end{tabular}

Estimated MDR-TB cases among notified TB cases

$\mathrm{n}^{\#} \quad \begin{gathered}\% \text { of cases by } \\ \text { country income } \\ \text { group }\end{gathered}$

group
Notified MDR-TB cases

n $\quad \begin{gathered}\% \text { of cases by } \\ \text { country income } \\ \text { group }\end{gathered}$
group

\begin{tabular}{|c|c|c|c|c|c|c|c|c|c|c|c|c|}
\hline Low & 9 & 30 & 30 & 30 & 26600 & 10 & 10 & 10 & 3161 & 4 & 4 & 4 \\
\hline Total & 30 & & & & 280000 & & & & 77956 & & & \\
\hline
\end{tabular}

Income groupings as per World Bank 2013 (discussed further in [17]). OECD: Organisation for Economic Co-operation and Development.

\#: rounded to two significant figures.

\section{Discussion}

Surveillance data from the 30 countries with the highest numbers of MDR-TB patients in the world indicate progressive improvements in the scaling up of MDR-TB case detection and enrolment on treatment during 2009-2012. However, they also show that countries are moving at varying speeds on their trajectory towards ensuring universal access to the different aspects of MDR-TB care. Overall, the testing of patients for resistance to rifampicin and isoniazid, the mainstay first-line drugs used in first-line anti-TB regimens, remains extremely low in new patients except in eastern European countries and Peru. Among previously treated patients, only Belarus and Kazakhstan are approaching $100 \%$ coverage. The global detection of MDR-TB is thus very incomplete, but detection has exceeded $70 \%$ of the estimated amount in Belarus, Kazakhstan, South Africa and Ukraine. DST for second-line drugs among confirmed MDR-TB cases remains relatively high. The rate of detection of MDR-TB cases has improved dramatically over the last few years in India, reaching $26 \%$ of the estimated caseload, and it has also been matched with a capacity to place diagnosed patients on appropriate treatment. Treatment success remains low in most countries; however, Bangladesh, Ethiopia, Kenya and Viet Nam (all heavily reliant on external aid to support MDR-TB control) have shown that it is possible to reach and surpass the $75 \%$ target under programmatic conditions.

The global burden of MDR-TB weighs heaviest in middle-income countries, and this is partially explained by the fact that the two countries with the highest population in the world, India and China, now belong to this income group. However, it is also likely that whilst there is good access to isoniazid and rifampicin in middle-income countries, this might not be matched by effective measures to ensure their rational use and to forestall the development of resistance. The quality of the medicines available may also be an issue. These factors may explain the high risk of MDR-TB among TB patients, particularly in countries of the former Soviet Union, where anti-TB drug shortages and a disruption in healthcare services characterised the early years following their independence over two decades ago [20]. It is also worth noting that the MDR-TB cases that occur in many high-income countries originate from individuals who migrate from these countries. The concentration of MDR-TB in middle-income countries is also an opportunity for targeted investments, both domestic and external. Many middle-income countries have a better developed health infrastructure and more skilled healthcare professionals than low-income countries; these are crucial elements when mounting complex interventions for MDR-TB care at a large scale. These countries may therefore be well positioned to reach high numbers of MDR-TB patients effectively and within a short time period. If external funding agencies aspire to reach out to large numbers of MDR-TB patients and secure faster returns for the resources invested, it is more likely that they can do this effectively by targeting middle-income countries. For this to happen, agencies may have to review the eligibility criteria that they apply when selecting countries to support and/or focus on the provision of funding that can leverage larger domestic investments and policy changes that will enhance the rational use of drugs and the quality of care. It is also important that external investment does not displace domestic funding or the capacity to increase domestic funding; such funding is crucial to ensure the sustainability of efforts into the future $[21,22]$.

Case detection remains low in many places as a result of challenges in providing adequate testing facilities. Nonetheless, the reports discussed in this article do indicate that if the right investments are made in 

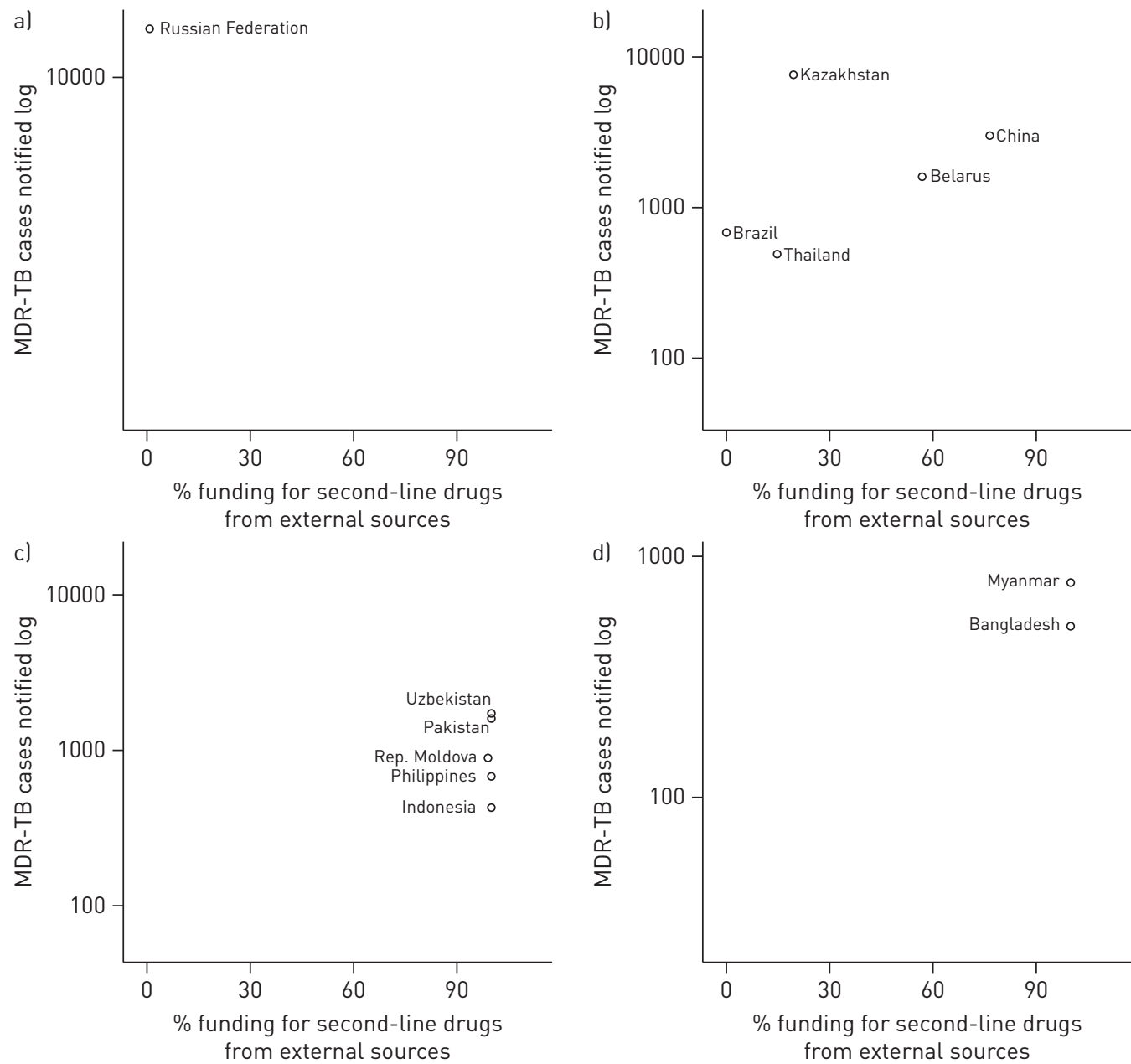

FIGURE 4 Multidrug-resistant tuberculosis (MDR-TB) notifications and percentage of funding received for second-line drugs from external sources, by income group, 2012. a) high-income group; b) upper middle-income group; c) lower middle-income group; d) low-income group. Only countries that reported data for both domestic and external funding are shown.

diagnostics, there is the potential for several countries to diagnose a large proportion of the MDR-TB caseload they are expected to find. This also extends to second-line testing in patients with MDR-TB for the detection of XDR-TB, which, although more frequent than first-line testing in countries reporting data, remains low overall.

There may be several reasons why the number of patients commencing MDR-TB treatment falls short of the total of patients identified as eligible for treatment: an inadequate supply of second-line drugs; patient death or loss to follow-up before initiation of treatment; other programmatic shortcomings. The data provided show that there is a substantial discrepancy in some countries and that this may even increase over time; in other countries it appears that enrolments outnumber the number of MDR-TB cases diagnosed. In addition to misalignment of diagnostic and curative services, a number of factors may affect the ratio of diagnosed MDR-TB cases to cases commencing treatment: there may be incomplete reporting of the detected cases (due to poor linkages between clinical and laboratory registers); patients placed on treatment are not enumerated in official statistics (as often happens when patients are treated in the private sector); the patients have commenced treatment without laboratory confirmation of MDR-TB; treatment began after the year of notification (this is accentuated when treatment programmes are catching up with the enrolment of a "backlog" of patients diagnosed in previous years).

In the reported data, distinct differences are seen between the countries with regard to the success rates for MDR-TB patients placed on treatment. Overall, the reporting of treatment outcome data remains incomplete, with substantial discrepancies between the number of patients for whom outcomes were reported and those originally notified. Some national programmes report remarkably high success rates: some of these cohorts consisted of a few patients treated under close monitoring in a small number of 
centres (such as in the Democratic Republic of Congo and Nigeria), although other small cohorts (particularly Papua New Guinea and Mozambique) had very poor outcomes as a result of high mortality or missing data. The differences in outcome between countries could be influenced by the patient mix (age profile, frequency of HIV co-infection (high in southern Africa) and resistance patterns) and treatment outcomes may not always be a direct reflection of the efforts of national programmes to treat their patients. The assignment of treatment outcomes should be reviewed carefully during monitoring and evaluation visits by programme supervisors to ensure that they are assigned appropriately and thus improve the quality of care. Several cohorts remain incomplete because of missing data: Brazil and Peru are examples of how reported treatment success can increase when data completeness is improved. It is also very important to ensure that the determinants of unfavourable outcomes are well known at programme level and that healthcare professionals are alerted to patients who are likely to interrupt treatment, die or not respond to treatment.

There are some limitations to our analyses, and these should be highlighted. Countries are responsible for the official data reported to WHO, and although checks for internal consistency are performed by WHO, these are not expected to address all possible sources of error. The MDR-TB burden estimates used depend on the completeness of overall $\mathrm{TB}$ notifications and tend to be conservative if the overall case detection of TB is low. Differences also exist in the accuracy of the data and how recently they were reported: we have used the latest available data, but in some countries a value based on modelling has been used in the absence of a direct measurement of the level of drug resistance among new and previously treated TB cases. Poor linkage of laboratory diagnostic data with surveillance data can also lead to odd results (e.g. DST coverage $>100 \%$ of cases in Kazakhstan). In addition, national surveillance programmes might not capture a sizeable proportion of TB and MDR-TB patients who are treated in the private sector. The assignment of treatment success without sufficiently discerning treatment failure may inflate figures showing favourable results, particularly in countries in which most cases that are declared to have a successful outcome have no bacteriological proof of resolution of disease, such as Ethiopia, the Democratic Republic of Congo and Papua New Guinea (fig. 3).

In conclusion, while a number of countries have made a huge effort to improve MDR-TB care in recent years, renewed vigour will be needed to increase the capacity to test, treat and ensure the best possible outcome for MDR-TB patients. Better access to improved diagnostics and WHO-approved molecular techniques is fundamental if we are to increase the rapid detection of MDR-TB. The capacity of healthcare workers to provide the required services, including the specialised care associated with MDR-TB, needs to be addressed through wider training, mentoring and learning tools, including e-Learning resources. Policies and practices to ensure that skilled healthcare personnel are retained are important given the specialised nature of the interventions required in the programmatic management of drug-resistant $\mathrm{TB}$ [23]. Models of care are required that put the emphasis on ambulatory care rather than hospitalisation. It is important to engage all relevant healthcare providers and partners in diagnosis, treatment and management of MDR-TB, employing an appropriate public-private mix mechanism, in order to improve universal access to MDR-TB care. The supply of second-line drugs, many of which provide a very poor market incentive for manufacturers, needs to be transformed and production needs to be diversified. The development of new drugs and shorter regimens are expected to improve the likelihood of curing MDR-TB patients, including in particular those infected with strains that have additional drug resistance [24-26]. Use of standardised monitoring methodology and wider employment of electronic data management is required for more accurate and complete information on the effectiveness of the global response. Finally, MDR-TB prevention remains one of the major cornerstones underpinning all TB control programmes. All of these principles are highlighted in the new global strategy for TB prevention, care and control after 2015, which was endorsed at the 67th WHA in May 2014 [27].

\section{References}

Global tuberculosis report. 2013. WHO/HTM/TB/2013.11. Geneva, World Health Organization, 2013.

2 Floyd K, Hutubessy R, Kliiman K, et al. Cost and cost-effectiveness of multidrug-resistant tuberculosis treatment in Estonia and Russia. Eur Respir J 2012; 40: 133-142.

3 Diel R, Vandeputte J, Vries G de, et al. Costs of tuberculosis disease in the European Union: a systematic analysis and cost calculation. Eur Respir J 2014; 43: 554-565.

4 Skrahina A, Hurevich H, Zalutskaya A, et al. Multidrug-resistant tuberculosis in Belarus: the size of the problem and associated risk factors. Bull World Health Organ 2013; 91: 36-45.

5 Ulmasova D, Uzakova G, Tillyashayhov M, et al. Multidrug-resistant tuberculosis in Uzbekistan: results of a nationwide survey, 2010 to 2011. Euro Surveill 2013; 18: 20609.

6 Zignol M, van Gemert W, Falzon D, et al. Surveillance of anti-tuberculosis drug resistance in the world: an updated analysis, 2007-2010. Bull World Health Organ 2012; 90: 111-119D.

7 Abubakar I, Zignol M, Falzon D, et al. Drug-resistant tuberculosis: time for visionary political leadership. Lancet Infect Dis 2013; 13: 529-539. 
Ahuja SD, Ashkin D, Avendano M, et al. Multidrug resistant pulmonary tuberculosis treatment regimens and patient outcomes: an individual patient data meta-analysis of 9,153 patients. PLoS Med 2012; 9: e1001300

9 Falzon D, Gandhi N, Migliori GB, et al. Resistance to fluoroquinolones and second-line injectable drugs: impact on MDR-TB outcomes. Eur Respir J 2013; 42: 156-168.

10 Migliori GB, Sotgiu G, Gandhi NR, et al. Drug resistance beyond extensively drug-resistant tuberculosis: individual patient data meta-analysis. Eur Respir J 2013; 42: 169-179.

11 WHO Global Task Force outlines measures to combat XDR-TB worldwide. http://www. who.int/mediacentre/ news/notes/2006/np29/en/ Date last updated: October 17, 2006. Date last accessed: June 30, 2014.

12 Sixty-second World Health Assembly. Resolutions and decisions; annexes. WHA62.15 Prevention and control of multidrug-resistant tuberculosis and extensively drug-resistant tuberculosis. http://apps.who.int/gb/ebwha/pdf_files/ WHA62-REC1/WHA62_REC1-en.pdf

13 The Global Planto Stop TB2011-2015: transforming the fight towards elimination of tuberculosis. WHO/HTM/ STB/2010.2. Geneva, World Health Organization, 2010.

14 Laserson KF, Thorpe LE, Leimane V, et al. Speaking the same language: treatment outcome definitions for multidrug-resistant tuberculosis. Int J Tuberc Lung Dis 2005; 9: 640-645.

15 Multidrug-resistant tuberculosis (MDR-TB) indicators. A minimum set of indicators for the programmatic management of MDR-TB in national tuberculosis control programmes. WHO/HTM/TB/2010.11. Geneva, World Health Organization, 2010.

16 Falzon D, Jaramillo E, Wares F, et al. Universal access to care for multidrug-resistant tuberculosis: an analysis of surveillance data. Lancet Infect Dis 2013; 13: 690-697.

17 World Development Indicators. http://data.worldbank.org/data-catalog/world-development-indicators Date last updated: July 22, 2014. Date last accessed: May 30, 2014

18 Wickham H. ggplot2: elegant graphics for data analysis. Springer, New York, 2009

19 R Project. R: a language and environment for statistical computing. Vienna, R Foundation for Statistical Computing, 2013.

20 Raviglione MC, Rieder HL, Styblo K, et al. Tuberculosis trends in eastern Europe and the former USSR. Tuber Lung Dis 1994; 75: 400-416.

21 Floyd K, Fitzpatrick C, Pantoja A, et al. Domestic and donor financing for tuberculosis care and control in low-income and middle-income countries: an analysis of trends, 2002-11, and requirements to meet 2015 targets. The Lancet Global Health 2013; 1: e105-e115.

22 Raviglione M, Uplekar M, Vincent C, et al. Rebalancing the global battle against tuberculosis. The Lancet Global Health 2014; 2: e71-e72.

23 National Institute for Health and Clinical Excellence. Tuberculosis: clinical diagnosis and management of tuberculosis, and measures for its prevention and control. NICE clinical guideline 117. Manchester, National Institute for Health and Clinical Excellence, 2011.

24 The use of bedaquiline in the treatment of multidrug-resistant tuberculosis. Interim policy guidance. WHO/HTM/ TB/2013.6. Geneva, World Health Organization, 2013.

25 European Medicines Agency. Human medicines: deltyba. http: //www.ema.europa.eu/docs/en_GB/document_ library/EPAR___Summary_for_the_public/human/002552/WC500166235.pdf Date last updated: April 2014. Date last accessed: June 30, 2014.

26 With 75\% of patients now enrolled in the STREAM MDR-TB trial, investigators meet to discuss progress and next steps. The Union. http://www.theunion.org/news-centre/news/with-75-of-patients-now-enrolled-in-the-streammdr-tb-trial-investigators-meet-to-discuss-progress-and-next-steps Date last updated: July 22, 2014. Date last accessed: August 26, 2014.

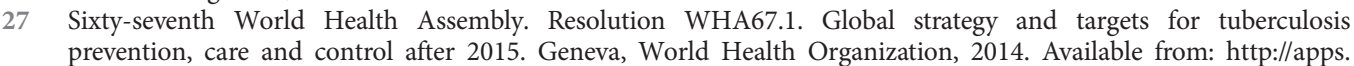
who.int/gb/ebwha/pdf_files/WHA67/A67_R1-en.pdf 